DOI: $10.1002 / \mathrm{smll} .200800604$

\section{Probe Tips Functionalized with Colloidal Nanocrystal Tetrapods for High-Resolution Atomic Force Microscopy Imaging ${ }^{\star \star}$}

\author{
Concetta Nobile, Paul D. Ashby, P. James Schuck, \\ Angela Fiore, Rosanna Mastria, Roberto Cingolani, \\ Liberato Manna, and Roman Krahne*
}

The performance and resolution of atomic force microscopy (AFM) imaging depends mainly on the quality and shape of the probe tip, since the obtained AFM image is a convolution of the tip profile and the sample structure. Therefore, tip radii that are smaller and aspect ratios that are higher than the sample features are desirable in order to obtain good images. Progress in the ability to design, fabricate, and assemble nanostructures in the size range of a few nanometers has raised the demand for probe tips with a corresponding resolution. Standard commercially available tips made of $\mathrm{Si}$ or $\mathrm{SiN}$ have a pyramidal shape with a tip radius of the order of $10 \mathrm{~nm}$ or larger and therefore do not image nanostructures with features in the few nanometer range adequately. One solution to this problem is the commercially available super-sharp Si probes with tip radius of $2 \mathrm{~nm}$, which, however, obtain their high resolution at a price: the sharp tip can break easily during an experiment. ${ }^{[1]}$ These limitations have stimulated many efforts to enhance the resolution of AFM by functionalizing the probe tips with high-aspect-ratio nanostructures. Carbon nanotubes have demonstrated excellent properties in this respect. ${ }^{[2]}$ Different approaches for the attachment of the carbon nanotubes to the AFM cantilever have been developed, ${ }^{[3-8]}$ and a spatial resolution of only a few nanometers has been demonstrated. ${ }^{[9]}$ However, the attachment of carbon nanotubes to the AFM tip is still a time consuming and very difficult task, and often results in non-reproducible nanotube configuration and placement. The optimal attachment geometry, with the tip perpendicular to the sample under investigation, is

[*] Dr. R. Krahne, C. Nobile, A. Fiore, R. Mastria, Prof. R. Cingolani, Dr. L. Manna National Nanotechnology Laboratory of CNR-INFM

Distretto Tecnologico ISUFI

Via per Arnesano, Lecce 73100 (Italy)

E-mail: roman.krahne@unile.it

Dr. P. D. Ashby, Dr. P. J. Schuck

Imaging Facility of the Molecular Foundry

Lawrence Berkeley Lab

1 Cyclotron Road, Berkeley CA 94720 (USA)

[**] The authors thank Benedetta Antonazzo for technical assistance in the Nanochemistry Laboratory. The work was supported by the SA-NANO European project (Contract No. STRP013698), the bilateral agreement with Berkeley (FIRB protocol RBIN048TSE) and work performed at the Molecular Foundry are supported by the Office of Science, Office of Basic Energy Sciences of the US Department of Energy under contract No. DE-AC0205CH11231.

口 Supporting Information is available on the WWW under http:// www.small-journal.com or from the author. particularly hard to realize. Also, the inherent thermal vibration of long nanotubes can cause difficulties when they are used for AFM imaging. ${ }^{[9,10]}$ Recent approaches to overcome these difficulties comprise the growth of multiwalled carbon nanotubes ${ }^{[11]}$ and the electron beam induced deposition of carbon nanocones on tipless cantilevers. ${ }^{[12,13]}$ For a recent review on AFM probes see elsewhere. ${ }^{[14]}$

Shape-controlled semiconductor nanocrystals are another very interesting family of nanostructures that can enhance the spatial resolution of AFM. Tetrapod-shaped nanocrystals are especially appealing for functionalizing AFM tips. Their ability to align on a surface with three supporting base arms, and the fourth arm pointing straight up, resembles an optimal geometry for the sensing of topography with the fourth, vertical arm. Recent advances in colloidal chemical synthesis have led to tetrapod samples with arm lengths of the order of several hundred nanometers and a diameter at the arm extremity well below $10 \mathrm{~nm}$. Moreover, the optoelectronic properties of shape-controlled nanocrystals can extend the functionality of AFM beyond the probing of topography. Banin and coworkers, for example, showed that AFM probes functionalized with spherical core/shell nanocrystals can be used for near field optical imaging. ${ }^{[15,16]}$

Here, we report the positioning of single CdTe tetrapods on flattened AFM tips and demonstrate the feasibility of these tips, via the vertical tetrapod arm, for high resolution AFM imaging. With our tip preparation we achieve an optimal probing angle of $90^{\circ}$, due to the use of contact mode scanning for the preparation of the tip flat. This inherently leads to a tip geometry with the flat parallel to the sample plane, which, combined with the capability of tetrapods to self-align with three arms contacting the surface and the fourth pointing vertically upward, results in a geometry where the vertical arm probes the topography at a $90^{\circ}$ angle to the sample surface. The high aspect ratio shape of the tetrapod arms, with diameters ranging from 5 to $10 \mathrm{~nm}$ and lengths ranging from 100 to $300 \mathrm{~nm}$, provides excellent properties for high-resolution topography scanning. In particular, we find that the tetrapod-functionalized tips work very well for imaging surfaces that are covered with nanocrystal samples. Furthermore, our tip fabrication technique could open the way for the fabrication of high aspect ratio optically and electronically sensitive probe tips due to the semiconductor properties of the tetrapods.

Large aspect ratio colloidal nanocrystal CdTe tetrapods with arm lengths ranging from 100 to $300 \mathrm{~nm}$ and diameters around $10 \mathrm{~nm}$ were fabricated by chemical synthesis as reported elsewhere ${ }^{[17]}$ and dissolved in toluene (see Supporting Information Fig. S2 for a TEM image of these very large tetrapods). The rapid growth of the tetrapod arms led to a pointed shape (i.e., to a decreasing arm diameter toward the arm extremity), which is advantageous for our purpose of high spatial resolution imaging (see Fig. 1b). Figure 1(b and c) show transmission electron microscopy (TEM) images of tetrapods deposited by drop casting onto a carbon coated TEM grid. The images show that the tetrapods self-align, with three arms contacting the substrate and the fourth arm pointing straight upward, appearing as a dark circular spot in the image.

A sketch of the tetrapod-functionalized AFM probe is shown in Figure 1a. 


\section{communications}
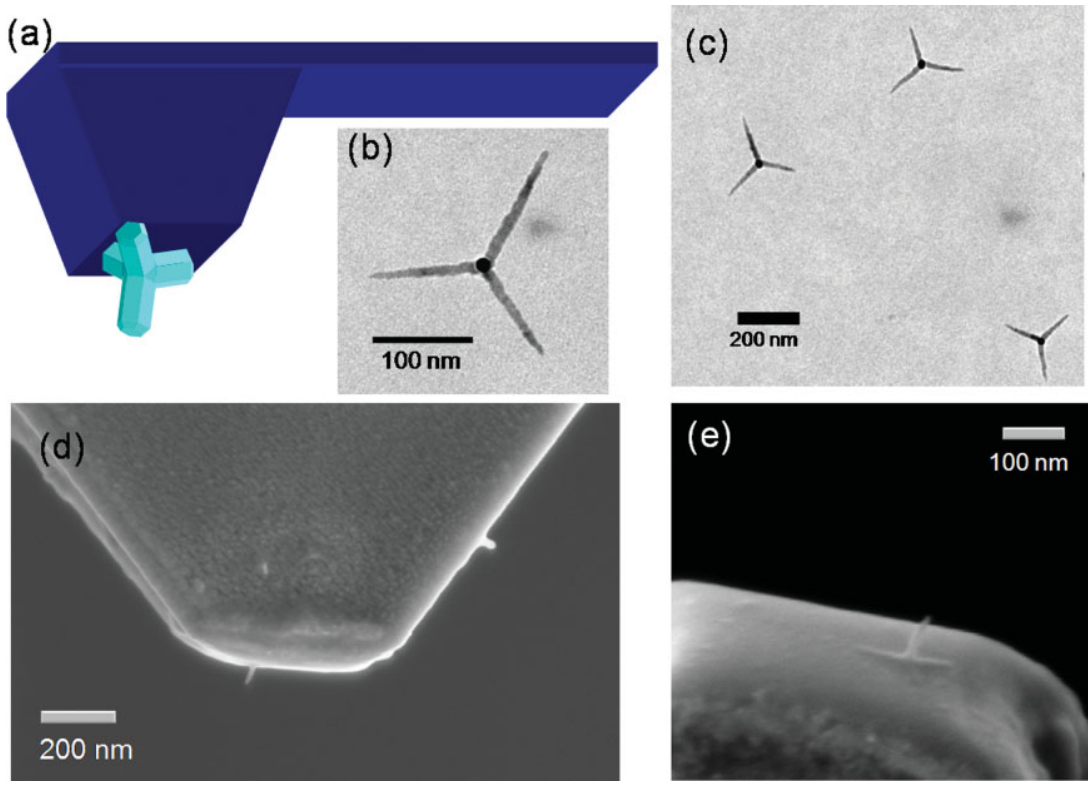

Figure 1. a) Schematic illustration of a tetrapod positioned on the tip of a flattened AFM probe (truncated pyramid and tetrapod dimensions are not to scale). b) and c) TEM images of CdTe tetrapods with arm length of $100 \mathrm{~nm}$. The tetrapods self-align and the arm pointing straight up appears in the image as a dark circular spot. d) and e) SEM images of a single CdTe tetrapod positioned on the flattened edge of an AFM tip.

The flat areas on the extremity of the AFM tips were obtained by scanning commercially available AFM tips ( $\mathrm{SiN}$ from Veeco) for approximately $1 \mathrm{~h}\left(\right.$ at $\left.60 \mu \mathrm{m} \mathrm{s}^{-1}\right)$ in contact mode on a diamond coated substrate (Nanoworld CDT coating). Then a thin metal layer of $\mathrm{Cr} / \mathrm{Au}(5 / 30 \mathrm{~nm})$ or $\mathrm{Pd}$ $(30 \mathrm{~nm})$ was evaporated on the tip side of the cantilever. The CdTe tetrapods dissolved in toluene were deposited on the flat tip edge from solution using a procedure similar to electrostatic trapping. In electrostatic trapping, the nanoparticles are polarized by an external field applied to an electrode structure, for example a structure of arrow-shaped nanoelectrodes, and are directed along the field gradient to the region of strongest electric field. This technique has been widely applied to position spherical nanoparticles between electrode pairs ${ }^{[18-21]}$ and can be readily extended to nanoparticles with more complex shapes like tetrapods, as shown in Figure 2(b and c). To attract the tetrapods to the tip of the flattened AFM probe we applied a voltage of $2 \mathrm{~V}$ to the metalized tip side of the cantilever. Figure 2a shows a calculation of the electric field strength (depicted by the density of the small red arrows) during the trapping in a dielectric medium, which illustrates that the electric field is strongest at the extremity of the pyramid (due to the "lightning-rod" effect ${ }^{[22]}$ ), and therefore the tetrapods are attracted to this region. The amount of tetrapods positioned on the tip flat can be controlled by the concentra- tion of tetrapods in the solution, and by the applied voltage and trapping time.

In detail, the flattened AFM probes were immersed in a diluted tetrapod solution (tetrapod concentration $7 \times 10^{-12} \mathrm{M}$ ) with the tip side up, and an AC voltage of $2 \mathrm{~V} \mathrm{rms}$ at a frequency of $50 \mathrm{kHz}$ was applied for $1 \mathrm{~min}$. The AFM probes were then taken out of the solution and dried by nitrogen flow. Finally, the tips were inspected by scanning electron microscopy (SEM) from different view angles, as displayed in Figure 1(d and e). We remark that the SEM images obtained from tilted angles (Fig. 1e and Supporting Information Fig. S3b) reveal that the three base arms of the tetrapod are in much closer contact with the surface than one would expect from an undistorted tetrahedron. Our AFM measurements of the height of the arm near the center exceed the arm diameter only by a few nanometers (see Supporting Information Fig. S3a) which suggests a strong distortion of the tetrapod, and indicates that the arm is in contact with the substrate over a large portion of its length. This effect was also observed by Fang et al. ${ }^{[23]}$ and demonstrates the strong interaction forces between the surface of the tip flat and the tetrapod.

The tetrapod-functionalized tips were mounted into a conventional AFM microscope (Molecular Imaging Pico Plus/ Veeco Multimode) and used to image different types of samples in AC or tapping mode under ambient conditions (see Supporting Information for more images). Figure 3 shows AFM images of CdTe tetrapods (same nanocrystal sample as displayed by TEM in Fig. 1[b and c]) deposited on an $\mathrm{Si} / \mathrm{SiO}_{2}$ surface by drop casting, which were imaged with the tetrapod-

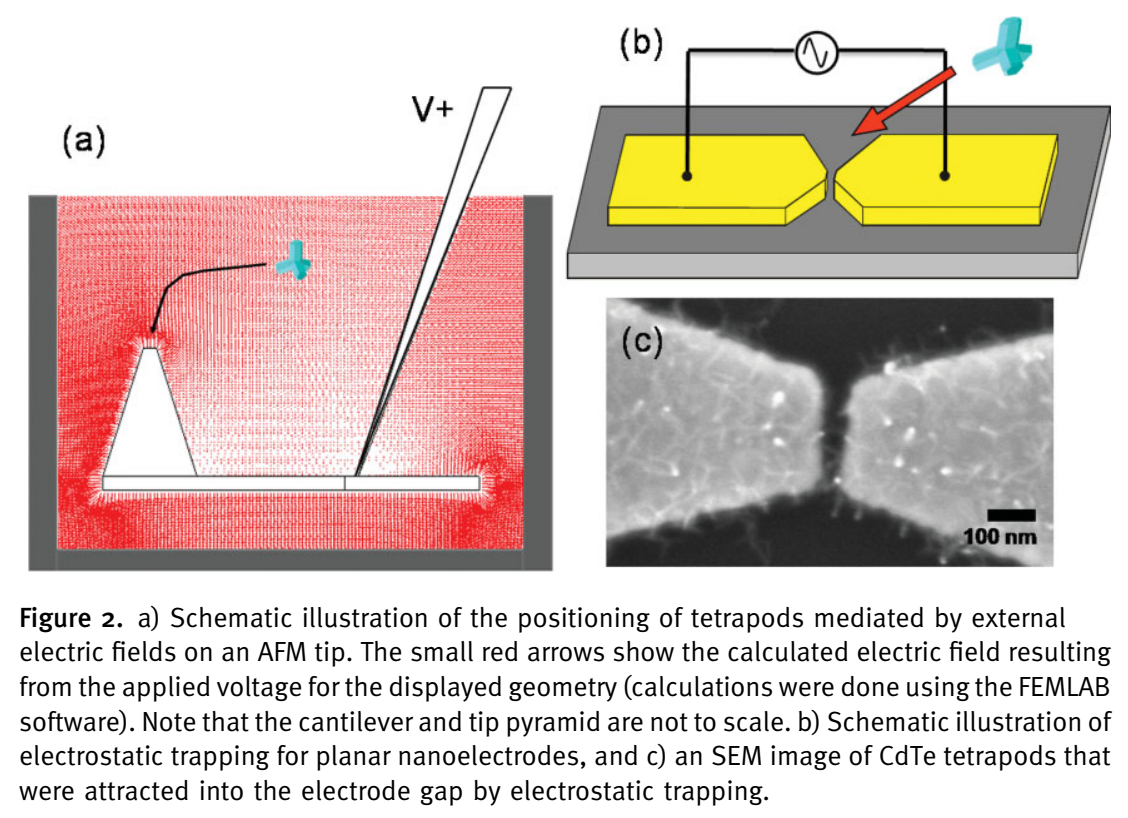


functionalized probe tips. The complex shape of the tetrapods is well resolved in the images, in particular the nearly vertical slope of the upwards-pointing arm. We obtained a maximum slope of $80 \%$ from the steep left and right sections of the vertical arm that are displayed in the inset of Figure 3a. Our tetrapod-functionalized tip was also capable of imaging the small variations in arm diameter on the three supporting arms that can be seen in the higher magnification TEM image displayed in Figure 1b. Profiles across the supporting tetrapod arms near the tetrapod center (see Supporting Information, Figure 3a) yield a maximum height around $15 \mathrm{~nm}$ and confirm

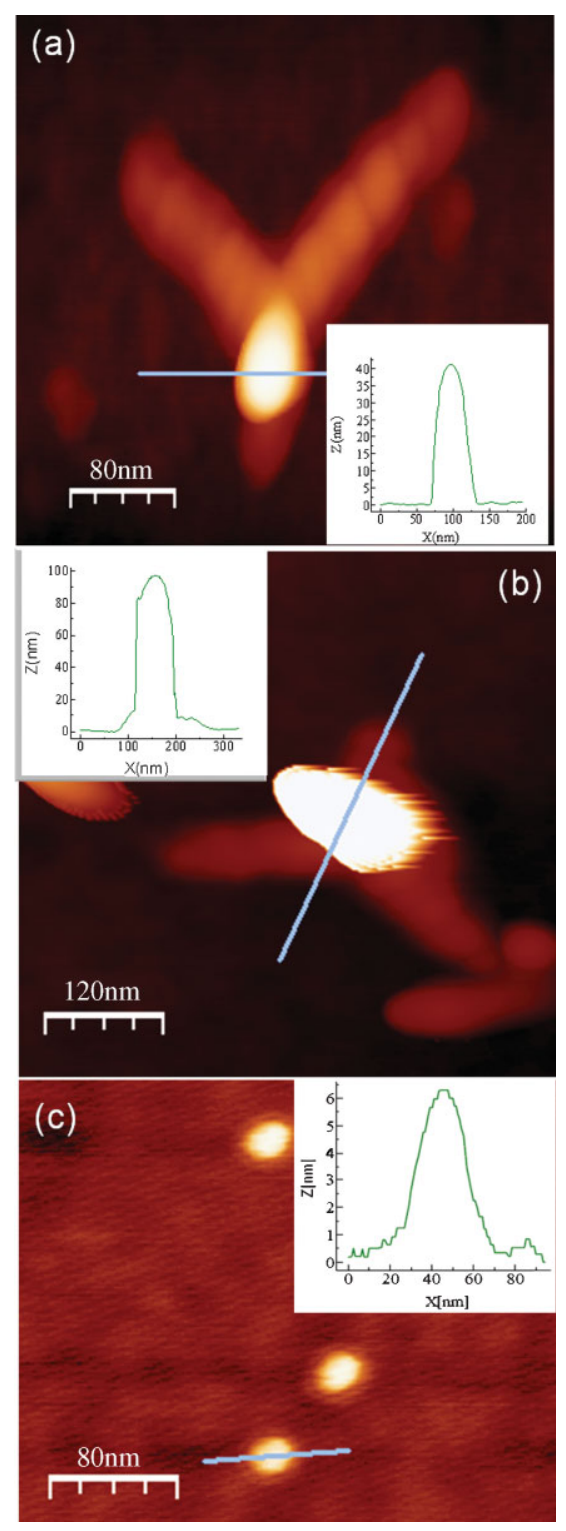

Figure 3. a) and b) Tapping mode AFM images of CdTe tetrapods deposited on an $\mathrm{Si} / \mathrm{SiO}_{2}$ surface obtained with tetrapod-functionalized probe tips. The width of the vertical arm imaged in (a) and (b) was 50 and $75 \mathrm{~nm}$, respectively. The maximum slope of $80 \%$ obtained with a tetrapod tip was obtained from the profile of the vertical arm of the imaged tetrapod in (a). c) Tapping mode AFM image of $\mathrm{CoPt}_{3}$ nanoparticles with $7 \mathrm{~nm}$ diameters (derived from TEM images) deposited on GaAs surface obtained with a tetrapod-functionalized probe tip. The insets show the topography profiles along the indicated sections. the strong attachment of these arms to the surface, as observed in the SEM images. The tetrapod in Figure 3a has a maximum height of $45 \mathrm{~nm}$, which indicates that the vertical arm broke during sample deposition. In an AFM study on similar CdTe tetrapods Fang et al. ${ }^{[23]}$ reported a considerable percentage of tetrapods with shortened vertical arms, and suggested that the damage was due to capillary forces during the drying process of the solvent. Figure $3 b$ shows a tetrapod with a maximum height of $100 \mathrm{~nm}$, and the nearly vertical topography of the arm pointing upwards is excellently imaged by our tetrapodfunctionalized tip (see line profile in the inset of Fig. 3b). The blurry tail of the vertical arm in the image is due to instabilities in the feedback control (similar behavior has been observed elsewhere ${ }^{[23]}$, which most probably results from attractive forces between the two vertical arms (from the imaging and the imaged tetrapod) during the scan. In order to estimate the resolution of our tetrapod-functionalized tips we imaged $\mathrm{CoPt}_{3}$ particles (shown in Fig. 3c), for which we obtained a diameter of $7 \mathrm{~nm}$ by TEM analysis. The full width of the line profile of a nanosphere in Figure $3 \mathrm{c}$ is $30 \mathrm{~nm}$. This leads to a tip radius of about $10 \mathrm{~nm}$, which is significantly larger than the $5 \mathrm{~nm}$ obtained from the TEM analysis of the tetrapod arms. This difference can be mainly attributed to the adjustments of the feedback circuit paramenters. We remark that such nanospheres deposited by drop casting are particularly difficult to image by AFM, since they get easily picked up or pushed around by the probe tip.

We find that the tetrapods on the functionalized tips are very robust. For example, Figure 4 shows an SEM image of the tip displayed in Figure 1d and e after scanning different samples for several hours (recording around 20 micron-size frames). The tetrapod is still in place and undamaged, and the vertical arm has maintained its original length. The strong attractive forces between the tetrapod and the flattened pyramid that are already present by drop casting the tetrapods from solution onto the tip are an advantage in this application. Also, the elasticity of the tetrapod arms (Fang et al. ${ }^{[23]}$ found elastic deformations of the tetrapods deposited on a plane substrate surface up to cantilever loads of $50 \mathrm{nN}$ ) prevents them from being easily damaged. We note that we did not observe the crashing of the tetrapod on the tip under normal non-contact and tapping mode scanning conditions, which would lead immediately to the complete loss of spatial resolution due to the flattened tip.

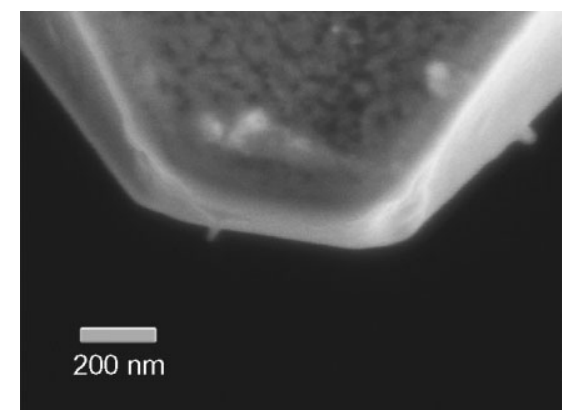

Figure 4. SEM image of the tetrapod tip after several hours of AFM imaging (about 20 frames of several micron size). 


\section{communications}

We remark that future advances in the synthesis of tetrapods will lead to better shape control, and in particular to tetrapods with longer arms. This will increase the performance of tetrapod-functionalized tips in terms of aspect ratio. In another direction, AFM probes that are functionalized with semiconductor nanocrystals can lead to applications that sense not only the topography, but also the optical or electronic properties of a sample.

In conclusion, we have demonstrated a quick and simple method to position single tetrapods at the extremity of flattened AFM tips. Such tetrapod-functionalized tips show very good AFM imaging performance with respect to spatial resolution and aspect ratio.

\section{Keywords:}

atomic force microscopy · functionalized tips - nanocrystals - tetrapods

[1] Y.-H. Shin, J.-W. Song, E.-S. Lee, C.-S. Han, Appl. Surf. Sci. 2007, 253(16), 6872.

[2] H. J. Dai, J. H. Hafner, A. G. Rinzler, D. T. Colbert, R. E. Smalley, Nature 1996, 384(6605), 147-150.

[3] S. S. Wong, A. T. Woolley, E. Joselevich, C. L. Cheung, C. M. Lieber, J. Am. Chem. Soc. 1998, 120(33), 8557-8558.

[4] J. H. Hafner, C. L. Cheung, C. M. Lieber, J. Am. Chem. Soc. 1999, 121(41), 9750-9751.

[5] J. H. Hafner, C. L. Cheung, C. M. Lieber, Nature 1999, 398(6730), 761-762.

[6] C. L. Cheung, J. H. Hafner, T. W. Odom, K. Kim, C. M. Lieber, Appl. Phys. Lett. 2000, 76(21), 3136-3138.

[7] A. T. Woolley, C. L. Cheung, J. H. Hafner, C. M. Lieber, Chem. Biol. 2000, 7(11), R193-R204.

[8] L. Chen, C. L. Cheung, P. D. Ashby, C. M. Lieber, Nano Lett. 2004, 4(9), 1725-1731.
[9] J. H. Hafner, C. L. Cheung, T. H. Oosterkamp, C. M. Lieber, J. Phy. Chem. B 2001, 105(4), 743-746.

[10] E. S. Snow, P. M. Campbell, J. P. Novak, Appl. Phys. Lett. 2002, 80(11), 2002-2004.

[11] C. V. Nguyen, R. M. D. Stevens, J. Barber, J. Han, M. Meyyappan, M. I. Sanchez, C. Larson, W. D. Ainsberg, Appl. Phys. Lett. 2002, 81, 901-903.

[12] I. C. Chen, L. H. Chen, C. Orme, A. Quist, R. Lal, S. H. Jin, Nanotechnology 2006, 17(17), 4322-4326.

[13] I. C. Chen, L. H. Chen, X. R. Ye, C. Daraio, S. Jin, C. A. Orme, A. Quist, R. Lal, Appl. Phys. Lett. 2006, 88(15), 153102.

[14] A. Yacoot, L. Koenders, J. Phys. D: Appl. Phys. 2008, 41(10), 103001.

[15] Y. Ebenstein, T. Mokari, U. Banin, J. Phy. Chem. B 2004, 108(1), 93-99.

[16] Y. Ebenstein, E. Yoskovitz, R. Costi, A. Aharoni, U. Banin, J. Phy. Chem. A 2006, 110(27), 8297-8303.

[17] L. Carbone, S. Kudera, E. Carlino, W. J. Parak, C. Giannini, R. Cingolani, L. Manna, J. Am. Chem. Soc. 2006, 128(3), 748-755.

[18] A. Bezryadin, C. Dekker, G. Schmid, Appl. Phys. Lett. 1997, 71(9), $1273-1275$.

[19] R. Krahne, A. Yacoby, H. Shtrikman, I. Bar-Joseph, T. Dadosh, J. Sperling, Appl. Phys. Lett. 2002, 81(4), 730-732.

[20] R. Krahne, T. Dadosh, Y. Gordin, A. Yacoby, H. Shtrikman, D. Mahalu, J. Sperling, I. Bar-Joseph, Phys. E-Low-Dimensional Syst. Nanostruct. 2003, 17(1-4), 498-502.

[21] T. Dadosh, Y. Gordin, R. Krahne, I. Khivrich, D. Mahalu, V. Frydman, J. Sperling, A. Yacoby, I. Bar-Joseph, Nature 2005, 436(7051), 677-680.

[22] J. D. Jackson, Classical Electrodynamics, 3rd ed., Wiley, New York 1999, p. xxi, 808.

[23] L. Fang, J. Y. Park, Y. Cui, P. Alivisatos, J. Schrier, B. Lee, L. W. Wang, M. Salmeron, J. Chem. Phys. 2007, 127(18), 184704.

Received: April 28, 2008

Published online: November 4, 2008 